\title{
Meinungen abbilden
}

DIE LIKERT-SKALA Um persönliche Meinungen zu erheben, eignet sich ein Fragebogen mit einer Likert-Skala. Der Befragte kreuzt an, inwiefern er einem Item zustimmt oder es ablehnt. Üblich ist sowohl eine gerade Zahl an Antwortmöglichkeiten wie eine ungerade. Bei ersterer muss sich der Befragte für eine Tendenz entscheiden, bei letzterer kann er auch die neutrale Mitte wählen. Ein Überblick.
$\mathbf{R}$ ensis Likert (1903-1981) war ein USamerikanischer Sozialforscher. 1932 hat er die nach ihm benannte Messtechnik „LikertSkala“ entwickelt. Mithilfe dieser Skala kann man in einem Fragebogen persönliche Einstellungen erheben (๑ Tab.) [1].

\section{Zustimmung oder Ablehnung erfassen >} Eine Likert-Skala ist eine Rating-Skala, mittels derer ein Befragter einem Statement zustimmt oder es ablehnt. Da diese sogenannte Intervallskala mehrstufig ist, muss die befragte Person nicht nur mit „ja“ oder „nein“ antworten, sondern kann eine abgestufte Antwort geben (॰ „Glossar“).

Die Zahl der Abstufungen kann variieren. In der Regel besteht eine Likert-Skala aus fünf, sieben oder elf Stufen. Das heißt, die Befragten können zwischen fünf, sieben oder elf Antwortmöglichkeiten wählen und einem Item mehr oder weniger extrem zustimmen bzw. es ablehnen. Häufig reicht die Likert-Skala von „Ich stimme völlig zu“ über die unentschiedene Haltung bis hin zu „Ich lehne völlig ab“. Das heißt, die mittlere Antwort - bei einer fünfstufigen Skala wäre das die drei steht stellvertretend für die Antwort „teils/ teils“ (๑ Tab.). Möglich ist aber auch, dass lediglich die Endpunkte mit einem verbalen Wert versehen sind [2, 3].

Jede Antwort will gut überlegt sein > Ein Fragebogen mit Likert-Skala sollte aus positiv und negativ formulierten Items bestehen, die beispielsweise Zustimmung und Gleichgültigkeit ausdrücken (॰ Tab.). Das bewirkt, dass die Befragten jedes Statement genau lesen und über die Antwort genau nachdenken müssen. Auf diese Weise vermeidet der Forscher, dass sie immer dieselbe Antwortkategorie ankreuzen. Ansonsten müsste er die Daten mit der sogenannten Ja-Sage-Tendenz aus der Auswertung ausschließen.

Häufig möchten Befragte keine eindeutig positive oder negative Meinung äußern und kreuzen daher gerne die neutrale Mitte an. Ein Forscher hat hier die Möglichkeit, die Befragten in der Einleitung aufzufordern, die neutrale Mitte möglichst nicht zu nutzen. Er kann sie aber auch einfach wegfallen lassen und die Befragten mit einer geraden Zahl an Antwortmöglichkeiten zu einer Meinung zwingen. Aber Vorsicht: Das kann dazu führen, dass sich die Anzahl fehlender Angaben (missing value) im Fragebogen erhöht, was wiederum die Qualität der Erhebung verschlechtert. Ein Forscher kann aber auch die Antwortformulierungen modifizieren (zum Beispiel: stimmt genau, stimmt ziemlich, stimmt wenig, stimmt nicht) bzw. lediglich die Endpunkte mit einer Textaussage versehen [3].

Vorsicht vor Fallen! > In der Regel sind bei diesem Fragebogenformat die Items und die entsprechenden Antwortmöglichkeiten gut verständlich. Allerdings reichen den Befragten die Antwortoptionen manchmal nicht aus bzw. sie stimmen nicht völlig mit ihnen über-

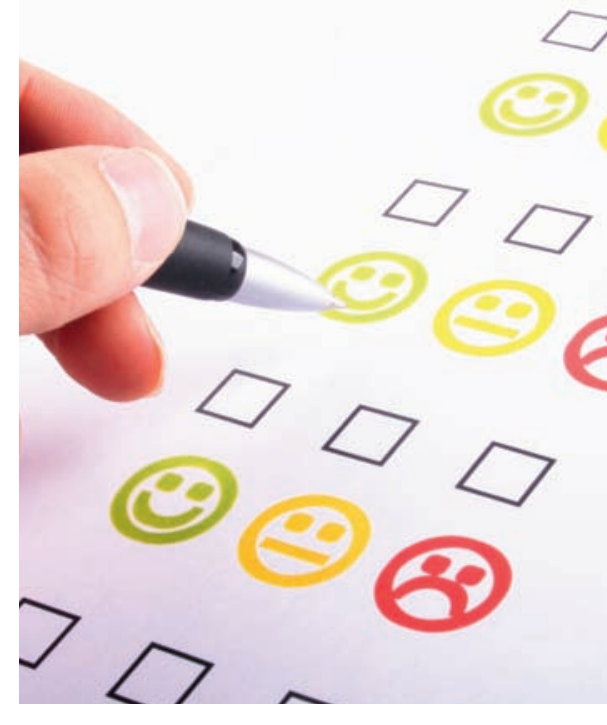

ein. Forscher sollten also die Antworten mit Bedacht auswählen. Eine weitere Stolperfalle: Befragte lassen sich häufig davon beeinflussen, wie sie vorangegangene Fragen beantwortet haben. Das heißt, falls sie bereits mehrmals zugestimmt haben, könnten sie versehentlich mit der Zustimmung fortfahren. Oder sie durchbrechen das Muster, wenn das Item sinnverdreht formuliert ist. Dann stimmen Befragte einem Statement zu, obwohl sie es normalerweise ablehnen würden.

Es gibt keine klare Regel, wie viele Antwortmöglichkeiten man den Befragten anbieten sollte. Denn eine ungerade Zahl erlaubt es ihnen, den neutralen Mittelweg zu gehen. Eine gerade Zahl hingegen zwingt sie dazu, sich zu entscheiden, auch wenn das nicht ihre wahre Position reflektiert. Trifft eine Zielgruppe ungern extreme Entscheidungen, spricht das eher für eine siebenstufige Skala als für eine fünfstufige. Auf diese Weise wäre für den Forscher dennoch eine Tendenz erkennbar.

Wichtig ist, dass alle Items eines Fragebogens eindimensional sind und das Gleiche messen, zum Beispiel „Klientenzentrierung“. Ist das nicht der Fall, sollte man die Items einzeln auswerten und eher von „Likert-skalierten Items" sprechen $[3,4]$.

Likert-Skalen in der Ergotherapie > Auch in ergotherapeutischen Assessments finden sich Likert-Skalen. Zum Beispiel im Test of Playfulness (ToP). Der Protokollbogen des Tests zur Spielfähigkeit beinhaltet beispiels- 


\section{Wissenschaft}

\section{Beispiel}

Angenommen, man will mittels Evaluationsbogen für Ergotherapeuten herausfinden, wie klientenzentriert sie sich und ihre Arbeit einschätzen. Dann könnten die Befragten den darin enthaltenen Items zustimmen oder sie ablehnen ( $\odot$ Tab.).

Um am Ende der Befragung einen Score errechnen zu können, ordnet man den Antwortkategorien jeweils einen Punktwert zu, zum Beispiel eins bis fünf. Ist ein Item negativ formuliert, bekommt es die umgekehrten Punktwerte (fünf bis eins) [1, 4].

Damit man später bei der Datenanalyse nicht die Übersicht verliert, sollte man die negativ formulierten Items wieder umpolen, damit alle Items die Ausprägung eins bis fünf aufweisen.

\section{Glossar}

Rating-Skala: Mithilfe einer Rating-Skala kann man abgestufte Aussagen erfassen. Sie besteht häufig aus sieben Schritten, deren Enden jeweils mit einer

Textaussage belegt sind, beispielsweise

„1: interessiert mich sehr" und „7: interessiert mich gar nicht“ [5].

Intervallskala: Eine Intervallskala gibt quantitative Werte wieder und unterteilt sich in gleich große Skalenabschnitte, zum Beispiel die Celsius-Skala, die hundert gleiche Gradabschnitte zwischen Gefrier- und Siedepunkt unterteilt [6].

Tab. Beispiel für eine fünfstufige Likert-Skala.

\begin{tabular}{|l|l|l|l|l|l|} 
& $\begin{array}{l}\text { ich stimme } \\
\text { völlig zu }\end{array}$ & $\begin{array}{l}\text { ich stimme } \\
\text { teilweise zu }\end{array}$ & teils/teils & $\begin{array}{l}\text { ich lehne } \\
\text { teilweise ab }\end{array}$ & $\begin{array}{l}\text { ich lehne } \\
\text { völlig ab }\end{array}$ \\
\hline $\begin{array}{l}\text { Ich lege großen Wert } \\
\text { darauf, die Therapieziele } \\
\text { gemeinsam mit meinem } \\
\text { Klienten zu formulieren. }\end{array}$ & & & & & \\
\hline $\begin{array}{l}\text { Es macht nichts, wenn } \\
\text { sich mein Klient im } \\
\text { therapeutischen Setting } \\
\text { nicht wohlfühlt. }\end{array}$ & 1 & 2 & 3 & 4 & 5 \\
\hline & 5 & & & & \\
\hline
\end{tabular}

weise die Items „engagiert sich aktiv“, „entscheidet, was getan werden muss“, „beteiligt sich am sozialen Spiel“. Für die Bewertung hat die Therapeutin vier Antwortmöglichkeiten: große, mittlere, geringe und keine Fertigkeit. Auch der Short Form 36 Health Survey Questionnaire (SF-36) arbeitet mit einer Likert-Skala. Bei der Frage „Wie häufig haben Ihre körperliche Gesundheit oder seelischen Probleme in der vergangenen Woche Ihre Kontakte zu anderen Menschen beeinträchtigt?" lauten die fünf Antwortmöglichkeiten: immer, manchmal, nie, meistens, selten.

Da subjektive Meinungen besonders in der Ergotherapie eine große Rolle spielen - sowohl auf Behandlungs- als auch auf Forschungsebene-, sind Fragebögen mit einer Likert-Skala geeignete Erhebungsinstrumente. Die „philo- sophische“ Frage nach der „richtigen“ Antwortzahl sollte man - wie die ergotherapeutische Behandlung auch - im Sinne der Zielgruppe beantworten.

Simone Gritsch

\section{$\Rightarrow$ Das Literaturverzeichnis finden Sie im Internet unter www.thieme.de/ergo- online > ,ergopraxis“ > „Artikel“.}

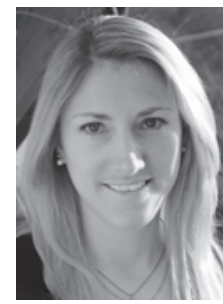

Simone Gritsch, Ergotherapeutin $\mathrm{BcOT}(\mathrm{NL})$ sammelte erste Erfahrungen mit Likert-Skalen in ihrer Bachelorarbeit. Heute befragt sie hin und wieder ergopraxis-Leser zu ihrer Meinung. 